\title{
Syntaxe du français médiéval et changement linguistique : Études de corpus
}

\author{
MONIQUE DUFRESNE \\ Université Queen's \\ dufresne@queensu.ca
}

et

MARIE LABELLE

Université du Québec à Montréal

labelle.marie@uqam.ca

Ce numéro spécial de la Revue canadienne de linguistique présente un ensemble d'études sur le français médiéval découlant d'un colloque qui a eu lieu à l'UQAM les 3 et 4 octobre 2014 sous le thème «L'utilisation des corpus (annotés) pour comprendre le changement linguistique ». Dans cette introduction, nous mettrons en évidence trois facteurs qui donnent à ce numéro son intérêt et son unité : les thèmes abordés, les approches linguistiques et sociolinguistiques et les modes d'utilisation des corpus.

\section{THÈMES}

Les articles se centrent sur trois grands thèmes qui marquent le changement historique du français : le développement des déterminants, la diffusion de la négation renforcée, et la structure de la phrase, plus particulièrement le rôle informationnel des éléments sur la syntaxe de la phrase et la structure $\mathrm{V} 2^{1}$ de la langue.

\section{1 Évolution des déterminants}

La langue latine classique ne connaissait pas de déterminants articles, alors que cette catégorie existe en français. Comment s'est effectuée cette transition qui s'est

\footnotetext{
${ }^{1}$ ACC: accusatif; ADJ: adjectif; ADVP: phrase adverbiale; AJ: auxiliaire avoir; CL: clitique; CP: phrase Comp; DIR: direction; DTV: datif; FinP: Phrase de finitude; IP: phrase; MAT: phrase matrice; NC: nom commun; NP: phrase nominale; NPR: nom propre; OV: objet-verbe; PON: ponctuation; PRN: incise; QUE: question; S: singulier; SBJ: sujet; SPE: discours direct; SUB: proposition subordonnée; SVO: sujet-verbe-objet; T: temps; V1: verbe premier; V2: verbe second; VJ: verbe lexical; VOC: vocatif; VPP: participe passé; WD: déterminant WH; WNP: syntagme nominal WH.
} 
déroulée en partie durant l'ancien français, état de langue qui permettait les noms nus («bare NPs ») dans beaucoup plus de contextes que le français moderne ? Deux articles se penchent sur cette question. Carlier et Lamiroy présentent une analyse qui traite de l'évolution de tous les déterminants par le biais d'études comparatives de traductions alignées. Elles comparent un texte latin de Cicéron à ses traductions en ancien français et en français moderne, ainsi qu'en espagnol et en italien. Elles proposent que l'évolution se déroule en cinq stades : 1) l'absence de déterminant article; 2) la prolifération de différents marqueurs de définitude en compétition; 3) la réduction du nombre de marqueurs à un seul article défini; 4) l'attraction d'autres marqueurs vers la même position; 5) la généralisation et la création de la catégorie des déterminants.

Poursuivant leurs études antérieures sur la disparition des noms nus, plus particulièrement d'un changement qui a affecté les déterminants articles au $12^{\mathrm{e}}$ siècle, à partir de deux textes anglo-normands, Déchaine, Dufresne, et Tremblay, pour leur part, développent une analyse en deux volets : nano-syntaxique et quantitative. Leur analyse nano-syntaxique se fonde sur une échine nominale qui comporte quatre têtes fonctionnelles, à savoir Nombre, Genre, D et Kas. Les conclusions de cette première partie ont été par la suite soumises à une analyse quantitative pour en valider les conclusions, selon lesquelles le genre des noms comptables joue un rôle dans l'expression du déterminant dans Le voyage de saint Brendan (ca. 11061121), mais non dans Les lais de Marie de France (ca. 1154-1189).

\subsection{Diffusion de la négation renforcée}

Donaldson s'intéresse pour sa part au cycle de Jespersen et, plus spécifiquement, à la généralisation de la négation renforcée. On sait qu'en ancien français, ne pouvait exprimer seul la négation. Avec le temps, ne a été renforcé de plus en plus souvent par un adverbe du type pas, point, mie. Cet adverbe, et plus spécifiquement pas, deviendra finalement partie intégrante d'une négation de phrase à deux termes ne...pas. Donaldson s'intéresse donc à la diffusion de la négation à deux termes, $\mathrm{au}$ détriment de la négation avec ne seul. Son étude est particulièrement intéressante pour son approche socio-historique, et nous y revenons ci-dessous dans la section 2 .

\subsection{La structure de la phrase}

Quatre articles tentent de déterminer si le rôle informationnel des éléments détermine leur réalisation dans la phrase, et si oui, de quelle façon. Qui plus est, deux de ces articles touchent de manière plus ou moins directe la question de la grammaire V2 de l'ancien français : l'ancien français avait-il une grammaire V2 et, si oui, comment a-t-il évolué vers une grammaire de type SVO ?

Tout d'abord dans le cadre de l'encodage informationnel des éléments de la phrase, Ingham s'intéresse au choix entre le sujet nul et le sujet pronominal postverbal. Il montre que ce choix n'est pas libre, mais déterminé par la nature informationnelle de l'élément préverbal. Lorsque celui-ci est D-linked, c'est-à-dire, qu'il constitue de l'information ancienne ou anaphoriquement reliée au discours précédent, le 
sujet est généralement pronominal; le sujet est généralement nul lorsque le constituent initial constitue une information nouvelle, donc, un focus informationnel.

De son côté, de Andrade étudie la disparition de l'ordre OV dans une perspective informationnelle et syntaxique, et montre, entre autres, que celle-ci s'est faite en trois temps : dans un premier temps, au $13^{\mathrm{e}}$ siècle, les noms nus et les topiques familiers ont cessés d'être encodés en position préverbale; les focus informationnels ont suivi au $14^{\mathrm{e}}$ siècle, et finalement, au $16^{\mathrm{e}}$ siècle, les topiques marqués et les focus contrastifs sont encodés, non plus dans la position initiale d'une structure V2, mais dans une position clivée ou de détachement à gauche.

L'approche standard de la structure V2 de l'ancien français est de considérer qu'il s'agit d'une langue ayant une grammaire du style de l'allemand, c'est-à-dire que le verbe se déplace dans la périphérie gauche de la phrase et qu'un unique constituant occupe une position sur sa gauche (voir entre autres Adams 1987, Roberts 1993, Vance 1997, Holmberg 2015). Cette approche a toutefois été mise en doute par divers chercheurs (notamment Kaiser 2000) sur la base de différences entre l'allemand et l'ancien français, et en particulier sur le fait que l'ancien français était moins strictement V2 que ne l'est l'allemand. Dans cette perspective, Rinke et Meisel (2009) proposaient que l'ancien français était une langue à topique initial.

Dans leur article, Labelle et Hirschbühler, poursuivant une discussion amorçée dans des études antérieures (Labelle et Hirschbühler 2013, 2014), argumentent que, du point de vue de l'encodage informationnel des éléments, l'ancien français est similaire à l'allemand, et concluent que l'hypothèse standard explique bien les données étudiées. Ils notent toutefois un changement dans l'encodage des éléments entre le $12^{\mathrm{e}}$ et le $13^{\mathrm{e}}$ siècle qui pourrait constituer le début du changement vers l'ordre SVO. Ils rejettent donc l'hypothèse que l'ancien français était une langue à topique initial plutôt qu'une langue à verbe second. En analysant les propositions matrices, ils montrent que, du point de vue de l'encodage informationnel (en termes de topique ou de focus) des éléments préverbaux et des sujets postverbaux, l'ancien français se comporte comme une langue V2.

Zaring, quant à elle, aborde la question du point de vue de l'ordre V2 en subordonnée, en se penchant sur l'inversion du sujet. Elle montre que les sujets pronominaux postverbaux, qui sont un indice du mouvement de verbe vers la périphérie gauche $(F i n P)$ de la phrase, sont extrêmement rares et ne se rencontrent que dans les propositions conjonctives. Par contre, l'inversion du sujet nominal est beaucoup plus fréquent et se trouve dans tous types de proposition. Elle propose de dériver cet ordre de mots à l'intérieur du domaine phrastique $(I P)$.

\section{APPROCHE LINGUISTIQUE VS SOCIOLINGUISTIQUE}

Tous les articles de ce numéro présentent des analyses quantitatives des données étudiées, et souvent des analyses statistiques. Trois articles (Ingham, de Andrade, et Labelle et Hirschbühler) font appel de manière plus ou moins élaborée à des analyses quantitatives contrastant un certain nombre de variables linguistiques. Il est de plus en plus incontournable de recourir en outre à des statistiques pour évaluer dans 
quelle mesure les différences observées peuvent être considérées comme significatives, d'autant que les moyens informatiques à la disposition des chercheurs permettent souvent d'extraire automatiquement un grand nombre de données se prêtant bien à des manipulations statistiques. À cet égard, on doit souligner le traitement statistique des données de la postposition du sujet nominal et pronominal effectué par Zaring, qui a eu recours à des analyses de régression utilisant comme facteurs la position du sujet, le type de proposition, le genre du texte, et la date du texte.

Deux des articles du présent numéro se démarquent en adoptant une méthodologie directement influencée par la sociolinguistique. Le changement linguistique implique nécessairement des phénomènes de variation, et par conséquent une dimension sociolinguistique. Or, les outils d'analyse développés en sociolinguistique ont été relativement peu transférés à l'étude de la linguistique historique. L'article de de Donaldson et celui de Déchaine, Dufresne, et Tremblay démontrent la faisabilité et l'intérêt d'aborder les données de ce point de vue.

Donaldson adopte une approche socio-historique (Romaine 1982) pour aborder la question de la diffusion de la négation renforcée en français. Il étudie trois variables : la nature de la proposition (principale vs subordonnée), l'oralité (récit vs oral représenté), et le sexe du locuteur dans le cas de l'oral représenté. Il montre que, dans les textes du $13^{\mathrm{e}}$ siècle étudiés (Queste 1225; Cassidorus 1267), la négation renforcée est significativement plus fréquente dans l'oral représenté que dans le récit, suggérant que la négation renforcée est, au départ, un phénomène lié à l'oralité; mais cette différence entre l'oral représenté et le récit ne vaut que pour les propositions principales, ce qui confirme les observations de Schøsler et Völker (2014) selon lesquelles la forme innovatrice (renforcée) s'est répandue dans les principales avant les subordonnées. La prévalence de la négation renforcée en principale se maintient dans les textes plus tardifs (la Vie de Saint Louis de Joinville (1308) et Jehan de Saintré (1456)), mais l'effet de registre n'y est plus présent, ce qui peut être associé à la diffusion de la forme renforcée. Pour ce qui est de la variable sexe, elle joue un rôle observable dans l'oral représenté dans la Quête, les hommes produisant plus de négation renforcée que les femmes, mais pas dans les textes postérieurs. Cela pourrait suggérer que le moteur du changement fut les hommes. Cette étude montre la faisabilité et la pertinence d'utiliser l'oral représenté, pour s'approcher de la langue orale, qui, bien sûr, n'est pas disponible pour les textes anciens, et pour étudier l'influence du sexe du locuteur sur son langage.

Déchaine, Dufresne, et Tremblay, pour leur part, ont recours à un logiciel conçu pour l'étude de la variation sociolinguistique, le logiciel GoldVarb. Elles l'utilisent pour raffiner leurs études antérieures (Dufresne et Tremblay 2012a, 2012b; Dufresne, Tremblay et Déchaine 2014) et pour calculer le poids respectif de différents facteurs linguistiques sur la réalisation du déterminant devant des noms comptables dans les deux textes d'anglo-normand qu'elles étudient. Leur analyse met en évidence un effet significatif de la définitude, du nombre et de la fonction grammaticale : la fonction sujet, le contexte défini et le singulier favorisent tous trois la présence d'un déterminant (par opposition à la fonction sujet, à l'indéfini et au pluriel qui favorisent les noms nus) dans les deux textes. Le genre du nom, par contre, ne joue pas le même rôle dans les deux textes et l'analyse quantitative 
révèle deux changements : le genre masculin comme facteur significatif pour les noms comptables, et le genre féminin pour les noms abstraits et de masse.

\section{3. ÉTUDES DE CORPUS}

De tout temps, les linguistes intéressés à l'ancienne langue ont travaillé à partir de corpus de textes. La méthode traditionnelle consistait à lire et à compter manuellement les occurrences de la construction visée. Cette méthode est extrêmement coûteuse en temps, sujette à des erreurs d'omission, et, dans certains cas, à des incohérences dans l'analyse des cas moins clairs. Avec le développement de corpus électroniques et d'outils de recherche appropriés, la recherche des données est de plus en plus facile, rapide, et systématique. L'intérêt et l'originalité du présent numéro résident dans la nature des corpus utilisés et dans leur utilisation pour aborder le changement linguistique.

\subsection{Les corpus annotés syntaxiquement}

L'utilisation de corpus électroniques annotés syntaxiquement a pris un essor important sous la gouverne d'Anthony Kroch (Université de Pennsylvanie) qui, avec ses collaborateurs, a développé un ensemble de corpus anglais (Kroch et Taylor 2000; Taylor et al. 2003; Kroch, et al. 2004a, 2004b; Taylor et al. 2006; Kroch et al. 2010). ${ }^{2}$ Dans la foulée de ce travail sur l'anglais et à l'instigation d'Anthony Kroch, des corpus annotés syntaxiquement s'élaborent graduellement pour d'autres langues : pour le français (corpus MCVF - Modéliser le changement: les voies du français, Martineau et al. $2010^{3}$ - environ 1 million de mots; et le Penn Supplement to the MCVF Corpus of Historical French, Kroch et Santorini, 2012 environ 150,000 mots); pour le portugais (Tycho Brahe Parsed Corpus of Historical Portuguese, Galves et Faria $2010 ;{ }^{4}$ WOChWEL $^{5}$ et CORDIAL-SIN, ${ }^{6}$ Ana Maria Martins et al.); pour l'islandais (Icelandic Parsed Historical Corpus, Wallenberg et al., $2011^{7}$ ) et pour le japonais moderne (NINJAL parsed corpus of modern Japanese, Prashant Pardeshi ${ }^{8}$ ).

Un corpus annoté syntaxiquement est un ensemble de textes dont les phrases sont entièrement analysées. L'exemple (2), tiré du corpus MCVF, illustre l'analyse de la phrase (1). On y voit, en plus des annotations de structure (CP, IP, NP, ADJ, ADVP), des annotation indiquant, entre autres, la différence entre proposition matrice (-MAT) et subordonnée (-SUB), le type de proposition (question =-QUE),

\footnotetext{
${ }^{2}$ Pour détails, voir : <http://www.ling.upenn.edu/hist-corpora>.

${ }^{3}<$ http://www.voies.uottawa.ca/voies_fr.html>

${ }^{4}<$ http://www.tycho.iel.unicamp.br/ tycho/corpus/en/index.html>

${ }^{5}<$ http://alfclul.clul.ul.pt/wochwel/index.html>

${ }^{6}<$ http://www.clul.ul.pt/pt/recursos/212-cordial-sin-syntax-oriented-corpus-of-portuguesedialects>

${ }^{7}<$ http://linguist.is/icelandic_treebank/Icelandic_Parsed_Historical_Corpus_(IcePaHC)>

${ }^{8}<$ http://npcmj.ninjal.ac.jp/?lang $=$ en>
} 
le discours direct (-SPE), les incises (PRN), la fonction des groupes nominaux (sujet $=\mathrm{SBJ}$, accusatif $=\mathrm{ACC}$, datif $=\mathrm{DTV})$, la différence entre verbe lexical $(\mathrm{VJ})$, verbe auxiliaire $($ avoir $=\mathrm{AJ})$, et participe passé $(\mathrm{VPP})$, la différence entre noms propres (NPR) et noms communs (NC) et leur nombre (singulier S), les clitiques (CL-), et la ponctuation $(\mathrm{PON})$ :

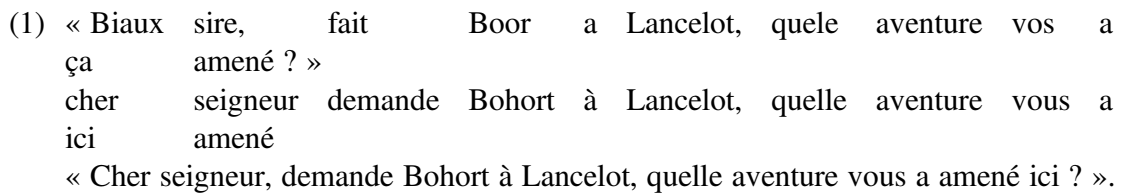
(La Queste del Saint Graal, p. 2)

(2) ((CP-QUE-MAT-SPE $(\mathrm{CODE}<\mathrm{q}>)$

$(\mathrm{PON}<$ open-guillemets $>)$

(NP-VOC (ADJ Biaux) (NCS sire))

$(\mathrm{PON}$,

$(\mathrm{CODE}<\operatorname{lb} \$ \$>)$

(IP-MAT-PRN-YYY (VJ fait)

(NP-SBJ (NPRS Boort))

(NP-DTV (DAT a) (NPRS Lancelot)))

$(\mathrm{PON}$,

(WNP-1 (WD quele) (NCS aventure))

(IP-SUB (NP-SBJ *T*-1)

(CL-NP-ACC (CL vos))

$(\mathrm{AJ}$ a)

(ADVP-DIR (ADV ça))

(VPP amené))

(PONFP ?))

(ID 1225-QUESTE-p,1.62))

L'annotation est effectuée automatiquement et corrigée de manière partiellement automatique par la suite. Cette annotation détaillée permet d'effectuer des recherches, non seulement sur des mots, comme dans les corpus non annotés, mais également sur des catégories grammaticales et sur des structures syntaxiques. On peut par exemple retrouver automatiquement toutes les propositions avec sujet postverbal, ou tous les groupes nominaux sans déterminant dont la tête est un nom commun, ce qui serait impossible avec un autre type de corpus. Le logiciel Corpus Search (Randall 2005) permet aussi d'extraire les données, de les coder automatiquement et d'exporter les codes vers un tableur ou vers un logiciel de statistiques. De plus, en donnant accès à un grand nombre de données extractibles automatiquement, ce type de corpus permet de faire des recherches qu'il serait difficile d'envisager autrement. C'est ainsi que, par exemple, Kroch et Santorini (2014) ont pu étudier le déclin de l'ordre OV en français, avec des objets multi-mots compléments de verbes non conjugués, Labelle et Hirschbühler (2013, 2014) ont travaillé sur le déplacement stylistique à gauche en ancien et moyen français, et Simonenko et Hirschbühler (2011, 2012) se sont penchés sur le placement des clitiques dans les propositions V1. 
L'élaboration de corpus de langues diverses conçus avec une même méthodologie permet d'envisager non seulement des études historiques des langues individuelles, mais également des comparaisons entre langues. À titre d'exemple, Kroch (2012) a présenté une étude comparative de l'évolution de l'ordre des mots en anglais médiéval et en français médiéval. Une étude comparative de la construction de déplacement stylistique à gauche en ancien islandais et en ancien français permettrait de valider une proposition de Mathieu (2006, 2009) selon laquelle l'ancien français comportait une construction semblable à celle de l'islandais, possiblement sous l'influence de la présence viking en Normandie. On peut également envisager une comparaison de textes des $12^{\mathrm{e}}$ et $13^{\mathrm{e}}$ siècles écrits en anglais, en français continental et en anglo-normand. Il s'agit de développements très prometteurs tant pour la recherche sur l'évolution de la syntaxe de la langue que pour les études sur le contact des langues.

Le corpus d'ancien et de moyen français compte un peu plus d'un million de mots et couvre une période allant du $10^{\mathrm{e}}$ siècle au $16^{\mathrm{e}}$ siècle. Quatre articles du présent numéro illustrent des utilisations différentes de ce corpus. De Andrade base son analyse sur les propositions du corpus comportant l'ordre OV. Déchaine, Dufresne, et Tremblay ont extrait des deux textes qu'elles ont choisis les groupes nominaux sujets et objets avec et sans déterminant dont la tête est un nom commun, afin d'étudier les changements dans le paradigme des déterminants au $12^{\mathrm{e}}$ siècle. Labelle et Hirschbühler ont travaillé à partir des propositions principales de type V2 et V1 pour étudier la définitude des sujets postverbaux et comparer les types de catégories précédant le verbe dans les propositions V2. Finalement, Zaring a utilisé cette méthodologie pour retrouver les propositions subordonnées et coder la position et la nature nominale ou pronominale des sujets.

\subsection{Traductions alignées}

Les autres auteurs du présent numéro ont choisi d'élaborer leur propre corpus afin de répondre à leurs questions de recherche, et cela de manière souvent originale. Carlier et Lamiroy, en particulier, abordent la question de l'évolution des déterminants du latin au français par le biais de traductions. Elles comparent le texte de Cicéron De Inventione et ses traductions d'une part en ancien français et en français moderne, et d'autre part en italien et espagnol. L'alignement des propositions avec le texte latin permet de mettre en évidence les contextes où les déterminants sont introduits là où le latin avait des noms nus.

\section{Conclusion}

L'utilisation de corpus a toujours été au cour de la théorie du changement linguistique et particulièrement dans sa perspective historique, puisqu'il c'est la seule façon d'avoir accès aux données. Par ailleurs le développement de corpus sur support informatique a permis l'accès non seulement à une plus grande quantité de données, mais aussi à des textes enrichis au départ d'annotations morphologiques, et plus récemment d'annotations syntaxiques. Ces dernières permettent des études 
plus fines et mieux documentées qui mènent à une meilleure compréhension des mécanismes impliqués dans le changement linguistique. Ainsi, bien que la configuration V2 ait fait l'objet de nombreuses recherches, les études présentées ici développent des analyses qui tiennent à la fois de la structure syntaxique et de la structure informationnelle. De plus, elles permettent une meilleure compréhension de la progression du changement tout en offrant une description détaillée des éléments qui apparaissent en position préverbale. Ces remarques valent aussi pour les études sur les noms nus. Les facteurs qui influencent l'apparition de noms sans déterminant sont connus depuis longtemps, mais aucune grammaire ni aucune étude n'en avait documenté leur poids ou leur importance. Un corpus annoté, où il est possible de distinguer les prédicats des arguments et de facilement repérer les sujets et les objets, a permis de mieux comprendre le rôle de chacun des facteurs, et plus précisément le rôle de la fonction grammaticale et de la définitude dans l'évolution des noms nus en français.

Pour terminer, revenons sur la quantité de données qui devient accessible grâce aux corpus annotés. Il importe de souligner que l'accessibilité à des faits linguistiques en grand nombre accorde une plus grande légitimité à des études réalisées à partir d'états de langues pour lesquels nous n'avons plus de locuteurs, même s'il ne faut pas perdre de vue que les données historiques seront toujours incomplètes. De plus, la possibilité de réaliser des études statistiques plus rigoureuses valident et complètent les analyses théoriques. Elles peuvent aussi mener à revoir certaines conclusions, comme c'est le cas de l'article de Déchaine, Dufresne et Tremblay où l'analyse statistique a donné une image plus précise du rôle du genre dans le changement du paradigme des déterminants et de la régression des noms nus.

En terminant, nous tenons à remercier les participants du Colloque L'utilisation des corpus annotés pour comprendre le changement linguistique qui s'est tenu à l'Université du Québec à Montréal les 3 et 4 octobre 2014. Les discussions qui ont eu lieu au cours de ces journées ont été des plus enrichissantes pour chacun des auteurs de ce numéro spécial de la RCL. Nous tenons aussi à remercier les étudiantes et les étudiants qui nous ont aidées lors du colloque et en particulier celles qui ont procédé à la révision des articles; leur soutien nous a été précieux. Tous nos remerciements vont aussi à nos collègues qui ont généreusement accepté d'évaluer les articles présentés ici, Julie Auger (Université d'Indiana), Lieven Danckaert (Université de Louvain), Roland Hinterholzl (Université de Venise), Maj-Britt Mosegaard Hansen (Université de Manchester), Cecilia Poletto (Université de Padoue), Christine M. Salvesen (Université d'Oslo) et Michelle Troberg (Université de Toronto à Mississauga).

Finalement. merci à tous nos commanditaires. sans qui le colloque et ce numéro spécial sur la linguistique de corpus n'auraient pas vu le jour :

- Conseil de recherches en sciences humaines du Canada - programme Connexion (subvention 611-2014-0063)

- Faculté des sciences humaines de l'UQAM

- Institut des sciences cognitives de l'UQAM

- Département de linguistique, UQAM 
- Centre de recherches sur le langage, l'esprit et le cerveau, UQAM

- Département d'études françaises, Université Queen's

- Service de la recherche, Université Queen's.

\section{CORPUS LITTÉRAIRE}

La Queste del Saint Graal, éd. A. Pauphilet. Paris : Champion (1923).

\section{RÉFÉRENCES}

Adams, Marianne. 1987. From Old French to the theory of pro-drop. Natural Language and Linguistic Theory 5(1) : 1-32.

de Andrade, A. 2013. A emergência da construção de topicalização no português europeu [The emergence of the topicalization construction in European Portuguese]. Technical Report of Project. Nr. 2011/19235-2. São Paulo : Biblioteca Virtual da FAPESP.

Dufresne, Monique et Mireille Tremblay. 2012a. D'un système de déterminants nuls à celui de déterminants réalisés phonologiquement : le cas du français. Communication présentée au Colloque Diachro VI, Louvain.

Dufresne, Monique et Mireille Tremblay. 2012b. Distribution des déterminants nuls en français médiéval et changement morphologique. Communication présentée au Congrès de l'Association for French Language Studies, Newcastle.

Dufresne, Monique, Mireille Tremblay et Rose-Marie Déchaine. 2014. L'expression du déterminant au XII ${ }^{\mathrm{e}}$ siècle: étude comparative du Voyage de Saint Brendan et des Lais de Marie de France. Actes du $4^{e}$ congrès mondial de linguistique française. DOI: <https://doi.org/10.1051/shsconf/20140801309>

Galves, Charlotte, et Pablo Faria. 2010. Tycho Brahe Parsed Corpus of Historical Portuguese. $<$ http://www.tycho.iel.unicamp.br/ tycho/corpus/en/index.html.>

Holmberg, Anders. 2015. Verb Second. Dans Syntax - Theory and analysis. An International Handbook, volume I, sous la direction de Tibor Kiss et Artemis Alexiadou, 342-383. Berlin: Mouton De Gruyter.

Kaiser, Georg A. 2000. Dialect contact and language change: A case study on word order change in French. Working Papers in Multilingualism, Series B. Hamburg: Universität Hamburg.

Kroch, Anthony. 2012. The evolution of word order frequencies in medieval English and French. Communication présentée à Historical Corpora 2012, Frankfurt-am-Main. $<$ http://www.ling.upenn./ kroch/handouts/hessen12.pdf $>$

Kroch, Anthony, et Beatrice Santorini. 2012. Penn Supplement to the MCVF Corpus of Historical French. University of Pennsylvania. <http://www.ling.upenn.edu/hist-corpora/>

Kroch, Anthony et Beatrice Santorini. 2014. On the word order of Early Old French. Communication présentée à SinFonIJA 7, Graz, Austria. <http://www.ling.upenn.edu: $\sim$ kroch:handouts:sinfonija7.pdf>

Kroch, Anthony, Beatrice Santorini et Lauren Delfs. 2004a. Penn-Helsinki Parsed Corpus of Early Modern English. CD-ROM, $1^{\text {st }}$ edition. <http://www.ling.upenn.edu/hist-corpora/>

Kroch, Anthony, Beatrice Santorini et A. Diertani. 2004b. Penn-Helsinki Parsed Corpus of Early Modern English. (http://www.ling.upenn.edu/hist-corpora/PPCEME-RELEASE-2/ index.html) 
Kroch, Anthony, Beatrice Santorini et Ariel Diertani. 2010. Penn Parsed Corpus of Modern British English. <http://www.ling.upenn.edu/hist-corpora/PPCMBE-RELEASE-1/index. html>

Kroch, Anthony et Ann Taylor. 2000. Penn-Helsinki Parsed Corpus of Middle English, second edition. CD-ROM. <http://www.ling.upenn.edu/hist-corpora/>

Labelle, Marie, et Paul Hirschbühler. 2013. Leftward Stylistic Displacement (LSD) in Medieval French. Dans Micro-change and macro-change in diachronic syntax, sous la direction d'Éric Mathieu et Robert Truswell, 145-167. Oxford : Oxford University Press.

Labelle, Marie et Paul Hirschbühler. 2014. Déplacement stylistique à gauche en ancien et moyen français. Dans Corpus 13 : Eléments initiaux dans la phrase : approches intergenres et inter-langues, sous la direction de Shirley Carter-Thomas et Sophie Prévost, 191-219. <https://corpus.revues.org/>. <halshs-01465415>

Martineau, France, Paul Hirschbühler, Anthony Kroch et Yves-Charles Morin. 2010. Corpus MCVF (parsed corpus), Modéliser le changement : les voies du français, Département de français, Université d'Ottawa. CD-ROM.

Mathieu, Éric. 2006. Stylistic Fronting in Old French. Probus 18(2): 219-266.

Mathieu, Éric. 2009. On the Germanic properties of Old French. Dans Historical Syntax and Linguistic Theory, sous la direction de Paola Crisma et Giuseppe Longobardi, 344-357. Oxford : Oxford University Press.

Randall, Beth. 2005. CorpusSearch 2. <http://corpussearch.sourceforge.net>.

Rinke, Esther et Jürgen M. Meisel. 2009. Subject-inversion in Old French: Syntax and information structure. Dans Proceedings of the workshop Null -Subjects, Expletives, and Locatives in Romance, sous la direction de Georg A. Kaiser et Eva-Maria Remberger, 93-130. Universität Konstanz. <http://nbn-resolving.de/urn:nbn:de:bsz:352-opus-78604>

Roberts, Ian. 1993. Verbs and Diachronic Syntax. A Comparative History of English and French. Dordrecht: Kluwer.

Romaine, Suzanne. 1982. Socio-historical linguistics: Its status and methodology. Cambridge: Cambridge University Press.

Schøsler, Lene et Harald Völker. 2014. Intralinguistic and extralinguistic variation factors in Old French negation with ne- $\emptyset$, ne-mie, ne-pas and ne-point across different text types. Journal of French Langauge Studies 24(1), 127-153.

Simonenko, Alexandra et Paul Hirschbühler. 2011. Changes in clitic position in Old French V1 clauses: V movement vs tense dependency? Communication présentée au 13th Diachronic Generative Syntax Conference (DiGS 13). University of Pennsylvania.

Simonenko, Alexandra et Paul Hirschbühler. 2012. Placement de clitiques dans les propositions V1 et évolution de la structure de la proposition en ancien français. Dans Constructions en changement, sous la direction de Monique Dufresne, 11-53. Québec: Presses de L'Université Laval.

Taylor, Ann, Anthony Warner, Susan Pintzuk et Frank Beths. 2003. York-Toronto-Helsinki Parsed Corpus of Old English Prose, $1^{\text {st }}$ ed. Oxford Text Archive.

Taylor, Ann, Arja Nurmi, Anthony Warner, Susan Pintzuk et Terttu Nevalainen. 2006. Parsed Corpus of Early English Correspondence, $1^{\text {st }}$ ed. Oxford Text Archive.

Vance, Barbara S. 1997. Syntactic Change in Medieval French Verb-Second and Null Subjects. Dordrecht: Kluwer.

Wallenberg, Joel C., Anton Karl Ingason, Einar Freyr Sigurðsson et Eiríkur Rögnvaldsson. 2011. Icelandic Parsed Historical Corpus (IcePaHC). Version 0.9. < http://www.linguist.is/icelandic_treebank>. 